\title{
Magnet Reliability in the Fermilab Main Injector and Implications for the ILC
}

\author{
M. A. Tartaglia, J. Blowers, D. Capista, D. J. Harding, O. Kiemschies, S. Rahimzadeh-Kalaleh, J. C. Tompkins
}

\begin{abstract}
The International Linear Collider reference design requires over 13000 magnets, of approximately 135 styles, which must operate with very high reliability. The Fermilab Main Injector represents a modern machine with many conventional magnet styles, each of significant quantity, that has now accumulated many hundreds of magnet-years of operation. We review here the performance of the magnets built for this machine, assess their reliability and categorize the failure modes, and discuss implications for reliability of similar magnet styles expected to be used at the ILC.
\end{abstract}

Index Terms-Electromagnet Reliability

\section{INTRODUCTION}

$\mathrm{I}^{\mathrm{N}}$ $\mathrm{N}$ the recently completed report on the reference design of the International Linear Collider (ILC) one of the key points is the need for high availability, due to the very large number of components and complex systems [1]. For magnets, whose failure can stop the machine operation and take many hours to diagnose and repair, this translates into the need for high reliability, particularly for conventional electromagnets cooled by low conductivity water (LCW). This paper reports on magnet reliability in the Fermilab Main Injector (FMI), a modern accelerator with nine years of operating experience. Many styles of both new and refurbished magnets have been in service since the start of operation in September 1998, providing a statistically interesting base of data.

The design and construction of new magnets, and the preparation for re-use of older Main Ring (MR) dipole and quadrupole magnets, is described in detail in the FMI Technical Design Handbook [2]. Also included are seven water cooled quadrupoles (known as WQB) which replaced some original reworked magnets in May 2006 [3]. These magnets include water cooled magnets, as well as smaller aircooled trim and higher order corrector (HOC) styles, which typically operate at much lower voltage and current than the water cooled varieties.

Our primary interest is in the performance of newly designed and built magnets, in order to benchmark reliability results from modern design approaches and materials used,

Manuscript received August 29, 2007. This work was supported by the U.S. Department of Energy.

M. A. Tartaglia. J. Blowers, D. Capista, D. J. Harding, O. Kiemschies, and J. C. Tompkins are with the Fermi National Accelerator Laboratory, Batavia, IL 60510 USA (corresponding author: 630-840-3890; fax: 630-840-8079; e-mail: tartaglia@fnal.gov).

S. Rahimzadeh-Kalaleh is with Embry-Riddle Aeronautical University, Daytona Beach, FL 32114 USA. production and inspection techniques, and to understand operational considerations. We also obtained and report on information regarding reworked Main Ring magnets, where the fabrication methods and prior service history are less well understood. We consider only magnets within the FMI Ring, and associated beamlines $(8 \mathrm{GeV}, \mathrm{P} 150, \mathrm{~A} 150$, abort transfer lines, and short remnant Main Ring sections) that include new magnets, as described in [2].

A variety of sources were consulted and studied to ensure that we have a complete and consistent picture of the reliability situation in the FMI. Catalogs of failures have been maintained in the Fermilab Accelerator Division (AD) and Technical Divisions (TD) by key personnel responsible for device management. Interviews were conducted and discussions held with operations experts from FMI and associated machines, as well as with TD magnet scientists, engineers, and technicians involved in the magnet design, construction, and failure investigations. Database records searches were made of numerous electronic logbooks and device service records that have existed for the duration of the FMI operation.

\begin{tabular}{|c|c|c|c|c|}
\hline Style & Cooling & Designation & Number & $\begin{array}{c}\text { Magnet } \\
\text { Hours }\end{array}$ \\
\hline New Dipole & LCW & $\begin{array}{l}\text { IDA, IDB, } \\
\text { IDC, IDD }\end{array}$ & 344 & 26782464 \\
\hline New Quadrupole & LCW & $\begin{array}{c}\text { IQC, IQD, } \\
\text { WQB, 3Qxx }\end{array}$ & 108 & 7940232 \\
\hline New Lambertson & LCW & ILA & 14 & 1089984 \\
\hline New C-magnet & LCW & ICA & 11 & 856416 \\
\hline Rework Dipole & LCW & $\begin{array}{l}\text { BDM, DDM, } \\
\text { EPB, ODM }\end{array}$ & 220 & 17128320 \\
\hline Rework Quad & LCW & $\begin{array}{l}\text { IQB, BQA, } \\
\text { BQB, SQA }\end{array}$ & 233 & 18063672 \\
\hline New Trim, HOC & AIR & $\begin{array}{l}\text { IDH, IDV, } \\
\text { ISA }\end{array}$ & 316 & 24602496 \\
\hline $\begin{array}{c}\text { Rework Trim, } \\
\text { HOC }\end{array}$ & AIR & $\begin{array}{l}\text { Many Main } \\
\text { Ring types }\end{array}$ & 233 & 18140448 \\
\hline
\end{tabular}

\section{Magnet Statistics}

The number of magnets and integrated time in service for the FMI ring and beamlines are cataloged in Table I, where they are organized by magnet style and cooling type, and by "new" or "rework" design history. We define "magnet hours" to be the product of the number of magnets (of each style) times the total number of machine operating hours for that 
style. The study period extends from 13 September 1998 until 01 August 2007, during which time the efficiency of operation is estimated (from logged magnet current data) to be $76.8 \%$. We have not distinguished between "up time" and "down time" - magnet hours include the entire operating period.

In Table II we show the number (Num) of failures that have required magnets to be removed from the tunnel, and mean time between failure (MTBF) for these in magnet hours. The two types of failure that require a magnet change, listed in Table II and discussed later in more detail, are internal water leaks and electrical shorts. For the cases with small statistics, we include the Poisson 90\% confidence level (CL) upper limit on the number of such failures detected, and use them to calculate the $90 \%$ CL lower limit on MTBF.

\section{TABLE II MAGNET FAILURE STATISTICS}

\begin{tabular}{c|c|c|c|c}
\hline \hline Style & $\begin{array}{c}\text { Num } \\
\text { Leaks }\end{array}$ & $\begin{array}{c}\text { MTBF } \\
\text { Leaks } \\
\text { [hours] }\end{array}$ & $\begin{array}{c}\text { Num } \\
\text { Shorts } \\
\mathbf{( 9 0 \% U L )}\end{array}$ & $\begin{array}{c}\text { MTBF Shorts } \\
\text { (90\%LL) } \\
\text { [hours] }\end{array}$ \\
\hline New Dipole & 0 & & $0(2.3)$ & $(1.16 \mathrm{E}+07)$ \\
New Quad. & 1 & $7.94 \mathrm{E}+06$ & 4 & $1.99 \mathrm{E}+06$ \\
$(7.99)$ & $(9.94 \mathrm{E}+05)$ \\
New Lamb. & 0 & & $0(2.3)$ & $(4.74 \mathrm{E}+05)$ \\
New C-magnet & 0 & & $0(2.3)$ & $(3.72 \mathrm{E}+05)$ \\
\hline Rework Dipole & 0 & & 1 & $1.71 \mathrm{E}+07$ \\
Rework Quad. & 3 & $6.02 \mathrm{E}+06$ & 9 & $(4.40 \mathrm{E}+06)$ \\
\hline New Trim,HOC & N/A & & $0(2.3)$ & $(1.07 \mathrm{E}+07)$ \\
Rework Trim, & N/A & & $0(2.3)$ & $(7.89 \mathrm{E}+06)$ \\
HOC & & & & \\
\hline \hline
\end{tabular}

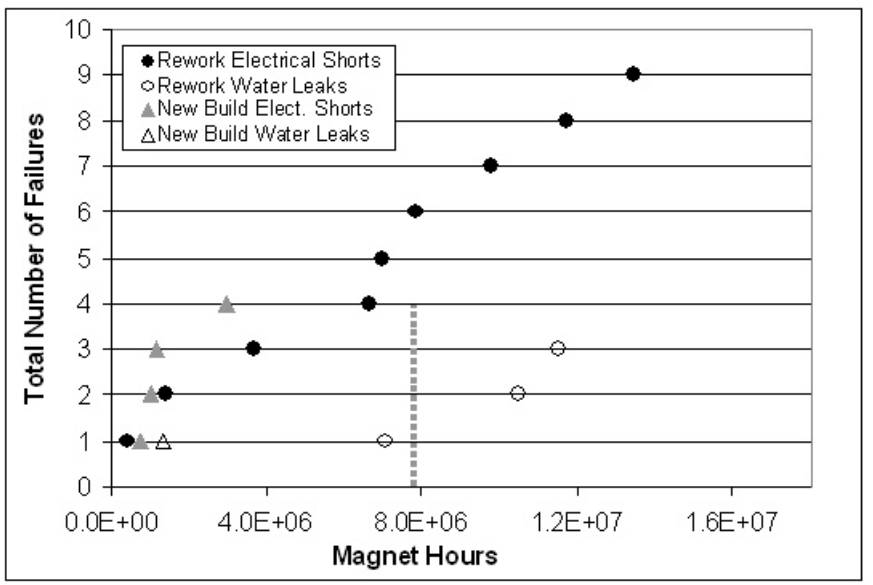

Fig. 1. History of quadrupole magnet failures in the Fermilab Main Injector. Dashed line shows newly built FMI quadrupole service time, and full scale shows the total number of magnet hours for rebuilt Main Ring quadrupoles.

The actual history of failures in these categories, from start of operations to present, is plotted in Fig. 1 separately for all newly constructed FMI quadrupoles and refurbished Main Ring quadrupoles. In this figure, the full time scale represents the total service history for the reworked Main Ring quadrupoles, while the newly designed and built FMI quadrupoles have experienced about half that, as shown by the vertical dashed line. Not shown in Fig. 1 is the single Main Ring dipole electrical failure, which occurred after 7.12E+06 integrated magnet hours of reworked dipole operation in the FMI.

\section{Discussion Of Failure Modes}

\section{A. Water Leaks}

Water leaks occur and are often recognized by trends and alarms that originate from sensors in the water system. They are also detected by vigilant inspection during tunnel access opportunities and repaired whenever possible. The decision to make repairs and what type of repair is often a judgment call, depending upon the severity of the leak and estimated time to repair. Typically these don't stop the machine - it is usually possible run until a more convenient time (e.g., maintenance period or shutdown) to repair or replace leaking devices. The risk is that continued exposure to water may result in a magnet electrical fault to ground, which does stop the machine.

Leaks have occurred predominantly at external braze joints, such as at manifolds, many of which are repaired in place. The impact of leaks at connection points has been reduced by intentionally locating water supply and return manifolds away from the magnets [4].

Magnets with internal leaks are ultimately replaced, and these are listed in Table II. Historical problems with internal braze joints led to great attention being paid to the multiple joints in the dipoles, including a conservative design, a reliable brazing technique (induction brazing), and rigorous inspection of each joint (ultrasound and helium leak check). Every attempt was made to avoid internal braze joints in the other magnets, and where necessary the design was conservative and the fabrication procedures were very explicit (with training and qualification required). No internal braze joint failures have occurred. All of the leaks that were detected and repaired have occurred at manifolds and tubing connections to coils (within the epoxy-potted ends).

Another potential failure is the bursting of the hoses connecting the magnets to the water headers. These are often anecdotally implicated as a source of magnet exposure to large volumes of water that can result in electrical failures: none have occurred (in FMI ring) during the machine operating life, even though the hoses are significantly past their five year rated lifetimes. This also implies that difficulties with hose degradation due to (hadronic) irradiation have not arisen.

\section{B. Electrical Shorts}

Electrical failures may be classified as shorts to ground ("ground faults") and turn-to-turn shorts. The latter are generally less of a problem, due to the much lower voltages involved. There has been only one recorded case of a turn-toturn short in the Main Injector, in a reworked MR quadrupole magnet. Log book notes must be read carefully, as the dominant locations of recorded ground faults are in power supplies and on the bus. We consider here only internal magnet problems, caused by failures of insulation, though we note that careful design of the magnet power connections can reduce the number of ground faults caused by external objects.

Shorts can, and usually do, stop the machine, if it is 
operating at the time of the failure. Since insulation failures can develop slowly over time, the main bussed magnet systems are subjected to a high voltage to ground (hipot) test at the beginning and end of each shutdown/maintenance access period to avoid a failure during operation. For the main dipoles and quadrupoles, this test is done at 1000 Volts; Aircooled trim and higher order corrector magnets are checked at low voltage after installation, but rarely thereafter.

One can imagine that similar procedures will be required to achieve high reliability in the ILC. It is not yet known what levels of voltage to ground must be tolerated by insulation in the ILC magnets, as most designs are still conceptual in nature. It is possible that they may require much less than the FMI 1000 Volt level, given that current ramps will be slow and the magnets are mostly powered individually - the majority in all areas in the RDR design - or in short strings, which could lead to increased reliability.

Ground faults on a bus require time and effort to localize, diagnose, and assess the problem; replace and align the failed device; and conduct checkout and startup procedures. Problem assessment typically includes calling in experts, conducting safe access procedures, and doing field diagnosis work. In 7 cases where it is clear that no other activities delayed startup of the machine (e.g., convenient time to make repairs on other systems), we calculated a mean time to repair (MTTR) for a magnet replacement in the FMI to be 18 hours.

The fraction of time a magnet is unavailable is MTTR/MTBF (for MTTR $<<\mathrm{MTBF}$ ), so reducing MTTR or increasing MTBF are equally effective ways to increase availability. A careful assessment of MTTR for ILC magnets has not yet been made, but will probably depend upon the area and magnet style. Individually powered magnets should make it faster to diagnose problems, but transportation of crews, equipment, and magnets over longer distances to the site of failure will tend to increase repair time in the ILC. Magnet sizes may be smaller in many cases, thus easier and faster to handle, than the FMI quadrupoles. Whereas FMI magnet changes break the beamline vacuum and require additional time to achieve operating vacuum conditions, ILC magnet designs should allow replacement without breaking vacuum.

\section{1) Analysis of New Quadrupole Failures}

The IQC and IQD quadrupoles were newly constructed for the FMI following quite closely the design of the Main Ring quadrupoles (IQB and $\mathrm{BQB}$ ) that were to be reused, with the functional difference being their lengths. In all these magnets the conductor was insulated with a pre-impregnated fiberglass tape and the coils were cured. The coil packages were then wrapped with dry fiber glass tape and installed in the magnet core. The whole assembly was vacuum impregnated with epoxy. This approach saves on vacuum impregnation tooling and generally provides a very robust package. In theory, the coils, tightly bonded to the core, do not expand on heating, so the epoxy is not stressed and does not crack even if it has lost some of its initial elasticity due to age, heat, or radiation.

Three early electrical failures of the new magnets led immediately to extensive study and magnet autopsies to determine the underlying cause, and the source of the problem quickly became evident. The new design sought to improve the reliability of these magnets by introducing a layer of insulating G10 between the coils and the steel core. This required a reduction in the thickness of the fiberglass tape insulation layer around coil conductors. The unanticipated consequence of the G10 was, in some cases, inadequate flow of the epoxy on both sides of the G10. These gaps translated into the lack of a good bond between the copper coil and the steel core. This is critical because when the magnet is energized the heated copper expands more rapidly than the steel core. With an integrated package, the coil is constrained against expansion, introducing a shear load on the epoxy. With a good epoxy bond, the load is distributed along the entire coil. However, the epoxy gaps and poor bond resulted in localized stresses at the ends of the magnets and thus cracks in the epoxy. Moisture eventually followed the cracks from the surface to the coil and a ground fault resulted.

A fourth new quadrupole magnet subsequently failed (in January 2002) and was determined to be of the same underlying cause. Since that time, no additional new quadrupole failures have occurred. While we cannot discount the possibility that additional magnets suffer from the disease of imperfect impregnation, perhaps to lesser extent, the statistics suggest that we may have passed the period of "infant mortality". It will require significant additional operating time to determine whether or not most of the newly fabricated quadrupoles suffer from the impregnation gaps as described above. Considering only operations since January 2002, the calculated $90 \%$ CL upper limit on the MTBF for newly built quads is slightly more than double the value in Table 1 , to $2.16^{*} 10^{6}$ hours.

In response to the findings, when the magnets were rebuilt the G10 insulation was eliminated and replaced by more layers of fiberglass tape. Subsequently, the insulation scheme has been changed radically and a number of new quadrupoles are being built with their coils vacuum impregnated independently from the core. The coils can expand without concentrated stress and the quality of the vacuum impregnation can be determined by visual inspection. This had been the approach for the Main Injector dipoles. The new wide aperture WQB quadrupoles also adopted this style, and there have been no failures of those 7 magnets in 15 months of operation.

\section{2) Discussion of Old Quadrupole Failures}

All of the Main Ring quadrupole magnets that were reused had been in service since the early 1970's, though ground fault failures over the years had led to a substantial number being rebuilt, a process that entailed burning off the epoxy, reinsulating the coils, and reimpregnating the magnet. The vacuum impregnation techniques had varied over the years, though mostly in the treatment of the magnet ends. We had anticipated rebuilding as many as $10 \%$ of the magnets for use in the Main Injector, but in fact all of them passed their electrical tests. The reworking for use in the Main Injector consisted of replacing the ceramic insulators which isolated the parallel water cooling circuits from the serially connected electrical circuit, along with some changes to the supports and vacuum tube.

While it could easily be a statistical fluctuation, it is interesting that there have been no additional reworked Main Ring quadrupole failures since April 2005. Unfortunately, 
there has not yet been an opportunity to evaluate the underlying reasons for failure of IQB and BQB magnets.

\section{Overheating}

Overheating of magnets can lead to degradation of epoxy insulation, failure of brazed joints, smoke, and even fire. A large temperature differential within a magnet, from inlet water temperature to outlet temperature, can produce large turn-to-turn stresses in the insulation and lead to cracking and electrical failures [5, 6]. High residual temperatures are also considered a personnel hazard when there is a need to access beam enclosures shortly after running. The smaller Main Injector magnets - trim dipoles and higher order correctors run at low enough power levels that air cooling is sufficient, and there have been no failures of those magnets. The main dipoles and quadrupoles are all water-cooled.

No evidence for blockages or degradation of cooling channels has yet been detected. In the FMI, the inlet temperature of the low conductivity cooling water (LCW) is held at $\sim 35^{\circ} \mathrm{C}$ (July) and the temperature rise is typically designed to be less than $\sim 5^{\circ} \mathrm{C}$. For the ILC, the Magnet Systems group has made a preliminary specification of an inlet temperature of $35^{\circ} \mathrm{C}$, and a maximum temperature rise of $25^{\circ} \mathrm{C}$; based upon FMI specifications, the ILC Conventional Facilities design is for a $10^{\circ} \mathrm{C}$ temperature rise.

It is also possible, and perhaps not uncommon, for magnets to be powered for short periods without LCW - for example, when water valves shut off for water work are not turned on until after a temperature alarm is detected. In fact, the insulation of one Main Injector quadrupole magnet was severely damaged when it was powered without LCW under just such a circumstance. Thermal switches are attached to all magnets in the FMI: they generate alarms but do not interlock to the power supplies (PS). In this case the alarm was not noticed until a smoke detector was triggered after several hours of running. We consider this to be an operations failure, rather than a magnet failure, so it is not included in the table.

For the ILC, this failure mode suggests that a minimum LCW flow should be a required condition to permit powering. This may imply a need for redundant flow sensors or switches, which may themselves be unreliable, to avoid false positive signals. Also, temperature switches ought to be interlocked to turn off the PS to protect magnets - this implies the need for careful quality control to verify that magnet interlock circuits are correctly wired to the power supply controls.

\section{CONCLUSION}

The combination of compromised insulation and water is especially potent. At typical operating voltages dry air is often a sufficient insulator even if a crack develops. All of the "magnet" failures that have caused the machine to stop working are associated with water. Anecdotally, in previous Fermilab accelerators, magnets would show a ground fault as soon as they were sprayed with water from themselves, an adjacent magnet, or another source. In the Main Injector, no failures have occurred in air cooled magnets - trim dipoles, quadrupoles, and higher order correctors - they are interspersed with water-cooled magnets, and local leaks can spray water on trim magnets as well as water-cooled devices, though perhaps with lower likelihood. The design of trims and correctors is simpler than water cooled magnets, they tend to run at lower currents and less thermal stress, and operate at much lower voltage to ground. In the FMI, loss of individual trim or corrector magnets (e.g., due to power supply failure) does not necessarily stop operation of the machine. We do not know to what extent this would be the case for the ILC.

The air-cooled magnets have been totally reliable - no failures result in a combined 90\% CL lower limit on MTBF of 18.6 million hours - as have been the newly designed dipoles (11.6 million hours). The dipoles benefited from the analysis of potential failure modes informed by Fermilab experience, an analysis that led to a conservative design and a rigorous quality assurance program. The demonstrated level of reliability achieved by these magnets is approaching the desired level for the ILC MTBF of $\sim 18$ million hours, suggesting that achieving this goal is possible with modern approaches. New FMI quadrupoles have averaged 2 million hours between failures, as have the reworked quadrupoles. Study of the failed new magnets concluded that unexpected stresses arose due to a well-intentioned design change and subsequent undetected manufacturing flaw. These failures happened early in the operating history, and it is possible that not all new quadrupoles suffered from this flaw. Additional running time without failures will increase our confidence in their reliability. Nevertheless, these failures highlight the reliability challenge facing the ILC, with an order of magnitude more magnets than in the FMI, and underscore the reality that it will take some years of operation before the results are known.

\section{REFERENCES}

[1] "International Linear Collider Reference Design Report 2007", ILCREPORT-2007-001, http://www.linearcollider.org/.

[2] Fermilab Main Injector Technical Design Handbook, August 1994, http://www-fmi.fnal.gov/fmiinternal/MI Technical_Design/index.html

[3] D.J. Harding, et al., "A Wide Aperture Quadrupole for the Fermilab Main Injector," Proceedings of the 2007 Particle Accelerator Conference, Albuquerque, NM.

[4] J. Satti, "The Fermilab Main Injector Dipole and Quadrupole Cooling Design and Bus Connections", Proceedings of the 1995 Particle Accelerator Conference, Dallas, TX.

[5] B.C. Brown, et al., "An Analysis of Fermilab Main Ring B3 Dipole Failures", December 30, 1993, Fermilab Report MTF-93-0004.

[6] B C. Brown, et al., "Report of the B2B3 Reliability Committee", April 9, 1994, Fermilab Report MTF-93-0009. 